\title{
Optical metrology applied in CCTV inspection in drain and sewer systems
}

\author{
Luís Martins ${ }^{1}$, Maria do Céu Almeida ${ }^{1}$, Álvaro Ribeiro ${ }^{1}$ \\ ${ }^{1}$ LNEC - National Laboratory for Civil Engineering, Avenida do Brasil 101, 1700-066 Lisbon, Portugal
}

\section{ABSTRACT}

This paper addresses the metrological quality of dimensional measurements based on images obtained from CCTV inspections in drain and sewer systems. In this type of indirect visual inspection, a significant number of absolute and relative dimensional quantities can be quantified, contributing to the characterization of the observations and, consequently, to the analysis of performance of drain and sewer systems outside buildings. Unfavorable environmental factors and conditions within the drain or sewer components affect estimation of the quantities of interest and the quality of the recorded images (lighting, lack of reference points, geometric irregularities and subjective assessments, among others). Quantification improvement of the dimensional quantities is a key objective to achieve a better assessment of components condition from these inspections. This study contributes to improve the quality of the dimensional measurements by defining experimental procedures, applicable to the optical systems used in CCTV inspection. The paper describes the European normative framework for these inspection activities and proposes approaches aiming at increasing confidence in the dimensional measurements based on the metrological characterization of the optical systems used, as well as, to establish a traceability chain. Results presented and discussed include the evaluation of measurement uncertainty.

\section{Section: RESEARCH PAPER}

Keywords: CCTV inspection; drain; sewer; dimensional measurement

Citation: Luís Martins, Maria do Céu Almeida, Álvaro Ribeiro, Optical metrology applied in CCTV inspection in drain and sewer systems, Acta IMEKO, vol. 9, no. 1, article 4, March 2020, identifier: IMEKO-ACTA-09 (2020)-01-04

Editor: Lorenzo Ciani, University of Florence, Italy

Received November 4, 2019; In final form January 30, 2020; Published March 2020

Copyright: This is an open-access article distributed under the terms of the Creative Commons Attribution 3.0 License, which permits unrestricted use, distribution, and reproduction in any medium, provided the original author and source are credited.

Funding: This work was supported by LNEC - National Laboratory for Civil Engineering, Portugal.

Corresponding author: Luís Martins, e-mail: Ifmartins@Inec.pt

\section{INTRODUCTION}

Drain and sewer systems are integrated in urban areas, often large and complex. These networks are composed by multiple types of components, such as drains and sewers, manholes, gullies, combined sewer overflows, storage and retention tanks, pumping stations, among others. These systems operate essentially under gravity to convey wastewater and stormwater to a treatment works or receiving water.

In the last decades, extensive work has been carried out by European standardization committees [1]-[3], aiming at the harmonization and improvement of strategic, policy and operational activities concerning drain and sewer systems. The established framework ambition is to contribute to fulfilment of strategic objectives of these services (including protection of public and occupational health and safety, environmental protection and sustainability). Set principles and functional requirements detailed in the standards are related to three main lines of action: (i) investigation and assessment; (ii) design and construction; and (iii) management and control.

The investigation and assessment of drain and sewer systems outside buildings [2] aims to establish an overview of their condition and performance and it can be defined as a sequential process, which includes: (i) purpose; (ii) scope; (iii) review of existing information; (iv) inventory update; and (v) hydraulic, environmental, structural and operational investigations. The results of these investigations allow determining the hydraulic performance, environmental impact, structural condition and operational deficiencies, supporting the comparison with the established performance requirements from which nonconformities can be found and management plans can be updated.

The investigations are carried out using several sources of information, including external or internal inspection activities for the detection and characterization of anomalies which can negatively affect the performance of the drain or sewer system. Close circuit television (CCTV) inspection is a largely used visual 
inspection technique for non-man entry components. The use of a remotely controlled CCTV camera is generally motivated by: (i) the need to inspect non-man entry components; (ii) safety issues, avoiding direct visual inspection by persons inside the drain and sewer systems; and (iii) faster and economic advantages, namely, when compared with quantitative methods such as laser scanning, ground piercing radar, sonar, infrared thermography and other available techniques [4]-[5].

This technique allows recording videos and individual images of the anomalies found in drains, sewers, manholes and inspection chambers. A standard European coding system has been developed [3], in line with existing national systems in member countries, facilitating common approaches and circulations of services within the EU. Data on observations includes: (i) type (e.g. fissure, deformation, displaced joints, defective connections, roots, infiltration, settled deposits, attached deposits and other obstacles, subsidence, defects in manholes and inspection chambers, mechanical damage or chemical attack); (ii) characterisation; (iii) location (longitudinal and radial); and (iv) quantification.

A significant number of absolute and relative dimensional quantities can be calculated, based on the recorded videos and images. Since these values are determining the results of the investigations and prioritization for corrective actions [6], efforts towards reduction of systematic deviations and measurement uncertainties can be highly relevant. In parallel with technological developments, on-going research efforts include automated image processing techniques [7] allowing the reduction of human influence in image analysis (which is time-consuming, prone to human error and to subjectivity) and the increase of image quality, e.g., by noise reduction [8]-[10]. However, from a metrological perspective, a deeper discussion on the measurement accuracy of the calculated quantities based on this method is still needed [11]-[13].

In the following sections, this paper presents constrains found to achieve an acceptable accurate level on dimensional measurements in CCTV inspections and proposes approaches for the improvement of the process and to the evaluation of measurement uncertainties. These approaches aim to reduce subjectivity in the image dimensional analysis and to increase reproducibility, using metrological characterization of the applied optical systems and its integration in a traceability chain.

\section{DIMENSIONAL MEASUREMENTS IN CCTV INSPECTIONS}

According to EN 13508-2:2003+A1:2011 standard [3], a number of quantities should be calculated and recorded to fully characterise the observation identified in the inspection.

In the case of absolute dimensions measurements (e.g. in millimetres or in degrees), record of the quantities that can be required include:

- width of fissures, cracks, connections, channels and sections;

- height of connections and channels;

- length of breaks, collapsed regions, intruding connections and sections;

- maximum dimension of obstacles;

- $\quad$ thickness of deposits;

- depth of sediments, anomalies in walls;

- $\quad$ longitudinal and radial displacement of joints;

- level difference between coverage and surface;

- curvature;

- angular displacement at joints.
In addition, relative quantities that can be required (expressed in percentage) include [3]:

- dimensional reduction;

- level relative to the diameter or vertical dimension;

- reduction in effective cross section area;

- intrusion length relative to the diameter or vertical dimension.

Examples of images obtained from a CCTV camera (e.g. Figure 1) in drain and sewer systems inspections are shown in Figure 2 and Figure 3, illustrating some common anomalies and the corresponding quantification parameters.

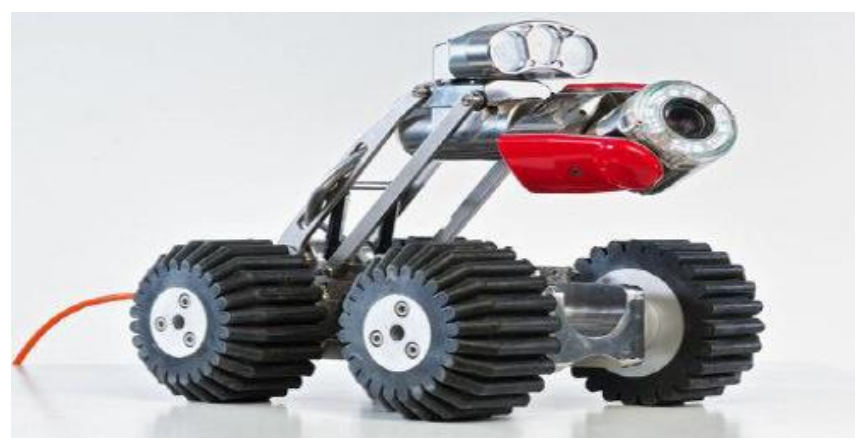

Figure 1. Remotely controlled CCTV camera used in drain and sewer systems inspections [14].

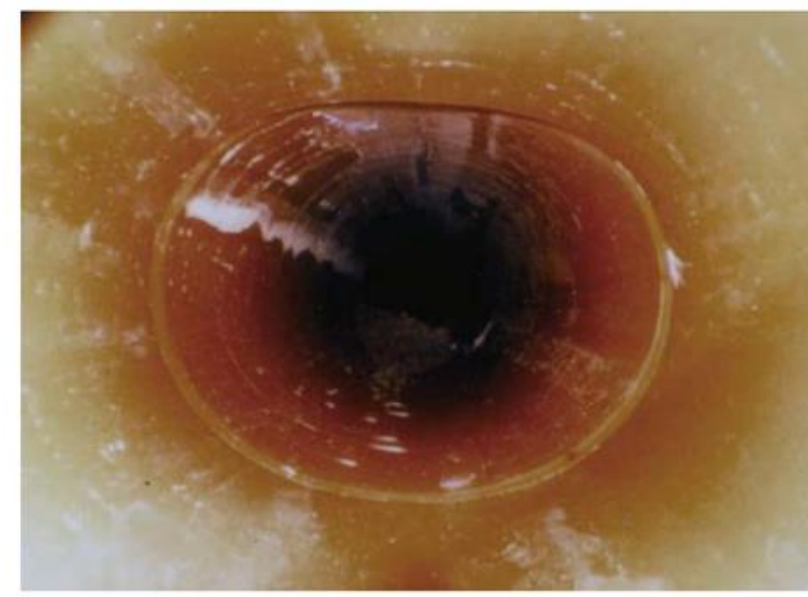

Figure 2. Inspection image showing dimensional reduction by deformation effect [14].

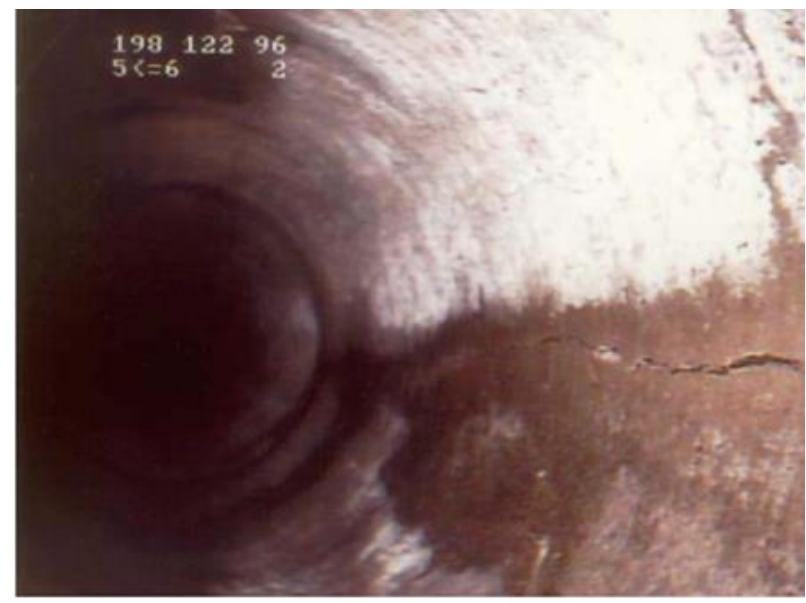

Figure 3. Inspection image showing fissure [14]. 
As a rule, in this type of inspection, the camera's longitudinal location in each observation is known and recorded with a recommended resolution of $100 \mathrm{~mm}$. An operational speed in the range of $0.1 \mathrm{~m} / \mathrm{s}$ to $0.2 \mathrm{~m} / \mathrm{s}$ is usually adopted in order to ensure an adequate detection of observations.

To improve the accuracy of the measurements, special attention was given to the CCTV camera position inside the sewers or drains, thus reducing geometrical projection deviations. An empirical tolerance up to $10 \%$ is usually defined for the CCTV camera vertical position $(1 / 2$ and $2 / 3$ of the drain/sewer height, for a circular/regular or egg shape, respectively).

Lighting tests should be carried out before each inspection, using image quality testing charts such as the Marconi Resolution Chart no. 1, allowing in situ qualitative evaluation of the recorded images, namely, the radiometric and spatial resolution as well as the geometrical distortion.

Although benefits arise from these operational practices, limitations on non-existence of reference points inside the component under inspection makes any dimensional measurement vulnerable to subjective image analysis. In the following section, approaches aiming at improving measurement accuracy are proposed, as well as, the comparability between measurements undertaken by different operators or in different moments in time.

\section{PROPOSED APPROACHES}

A first approach, aiming at increasing the confidence in the calculated dimensional quantities carried out, is to ensure the traceability of the metrological characterization of the optical system - the CCTV camera - used in drain or sewer inspection. This characterization includes both a geometrical component and a radiometric component, allowing a rigorous comparison between different CCTV cameras available in commercial solutions and their corresponding suitability to the measurement environment.

The main objective of the geometrical characterization is the quantification of CCTV camera intrinsic parameters such as the focal distance, the principal point coordinates and the lens distortion coefficients, which are input quantities for the determination of accurate dimensions in the field-of-view. This task can be achieved in a laboratorial setup, using traceable reference dimensional patterns and applying known algorithms such as the DLT - Direct Linear Transform [15], the Tsai [16] and the Zhang method [17].

The radiometric characterization can be carried out following the EMVA standard guidelines [18]-[19], allowing determining the CCTV camera sensitivity, linearity, noise, dark current, spatial non-uniformity and defecting pixels.

In addition to the CCTV camera metrological characterization, the measurement model itself must be defined and used in the dimensional measurement uncertainty evaluation, following the GUM framework [20]-[21].

If both the intrinsic (focal distance, principal point coordinates and lens distortion coefficients) and extrinsic parameters (camera position and orientation in the local or global coordinate system, acquired by dedicated instrumentation of the CCTV camera) are known, the perspective camera model [22], shown in Figure 4, can be used to determine the coordinates related to points of interest inside the sewer or drain. Assuming, in a first approach to this problem, that the lens distortion does not have a significant impact on the dimensional accuracy when compared

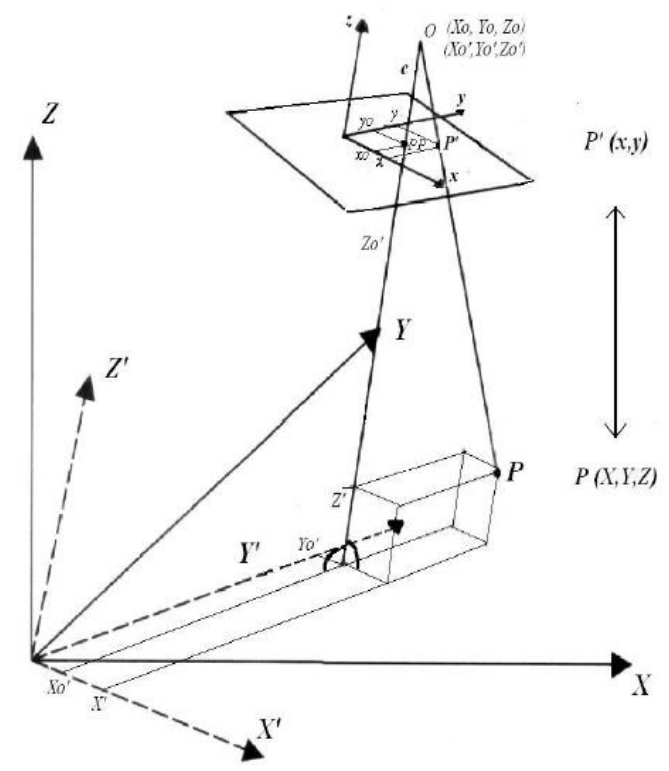

Figure 4. Schematic representation of the perspective camera model.

with other uncertainty components, the perspective camera model assumes a straight line between the interest point in global or local coordinate system $(X, Y, Z)$, where $Z$ defines the camera movement direction, and in the image $(x, y)$ coordinate systems, defining the so-called collinearity equations

$X=X_{0}+\left(Z-Z_{0}\right) \cdot \frac{r_{11} \cdot\left(x-x_{0}\right)+r_{12} \cdot\left(y-y_{0}\right)-r_{13} \cdot f}{r_{31} \cdot\left(x-x_{0}\right)+r_{32} \cdot\left(y-y_{0}\right)-r_{33} \cdot f}$

$Y=Y_{0}+\left(Z-Z_{0}\right) \cdot \frac{r_{21} \cdot\left(x-x_{0}\right)+r_{22} \cdot\left(y-y_{0}\right)-r_{23} \cdot f}{r_{31} \cdot\left(x-x_{0}\right)+r_{32} \cdot\left(y-y_{0}\right)-r_{33} \cdot f}$

where: (i) intrinsic parameters $-f$ is the focal distance and $\left(x_{0}, y_{0}\right)$ are the principal point image coordinates; (ii) extrinsic parameters $-\left(X_{0}, Y_{0}, Z_{0}\right)$ are the camera's global or local coordinates and $r_{i j}$ are the rotation matrix elements defined by the camera's orientation angles $\Omega, \Phi, K$, given by

$r_{11}=\cos \Phi \cdot \cos K$

$r_{12}=-\cos \Phi \cdot \sin K$

$r_{13}=\sin \Phi$

$r_{21}=\cos \Omega \cdot \sin K+\sin \Omega \cdot \sin \Phi \cdot \cos K$

$r_{22}=\cos \Omega \cdot \cos K-\sin \Omega \cdot \sin \Phi \cdot \sin K$

$r_{23}=-\sin \Omega \cdot \cos \Phi$

$r_{31}=\sin \Omega \cdot \sin K-\cos \Omega \cdot \sin \Phi \cdot \cos K$

$r_{32}=\sin \Omega \cdot \cos K+\cos \Omega \cdot \sin \Phi \cdot \sin K$

$r_{33}=\cos \Omega \cdot \cos \Phi$

If the camera's intrinsic and extrinsic parameters are not available, a less rigorous approach can be followed, assuming a parallel geometrical relation between the image plane and the cross-section plane in the drain or sewer and using an orthographic projection camera model [22] to define a scale coefficient, SC, between real dimension (in millimetres) and image dimension (in pixels). In the observed field-of-view, the only available dimensional reference is related to the height of the drain or sewer, $H$. Therefore, the dimensional quantification of a certain observation must be supported by the determination of the scale coefficient relative an average cross-section plane in the drain or sewer which includes the quantity to be measured and where its height is also visible, as shown in Figure 5. 


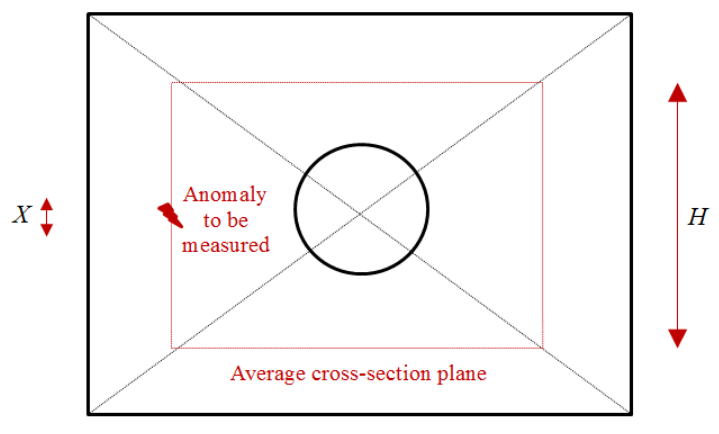

Figure 5. Schematic representation of the measurement procedure in CCTV camera images, based on the orthographic camera model.

In this approach, the image dimensions (in pixels) related to the drain or sewer height in the average cross-section plane, $p_{H}$, and to the observed anomaly, $p_{\mathrm{x}}$, are obtained by image processing. Therefore, the scale coefficient, expressed in millimetres per pixel, is obtained by

$S C=\frac{H}{p_{H}}$

while the dimensional measurement of the observation, $X$, (in millimetres) is given by

$X=S C \cdot p_{X}$

Since these equations can be considered as linear models, the application of the Uncertainty Propagation Law [20], results in the standard measurement uncertainty expressions for the scale coefficient, $u(S C)$, and for the dimensional measurement of the anomaly $u(X)$, respectively,

$u(S C)=\sqrt{\frac{1}{p_{H}^{2}} \cdot u^{2}(H)+\frac{H^{2}}{p_{H}^{4}} \cdot u^{2}\left(p_{H}\right)}$

$u(X)=\sqrt{p_{X}^{2} \cdot u^{2}(S C)+S C^{2} \cdot u^{2}\left(p_{X}\right)}$

where $u(H)$ is the standard uncertainty of the height of the drain or sewer and $u\left(p_{H}\right)$ and $u\left(p_{X}\right)$ represent respectively the standard uncertainties of the image dimensions of $p_{H}$ and $p_{X}$.

\section{RESULTS AND DISCUSSION}

This section contains the approach considered for the evaluation of the dimensional measurement uncertainty related to the adoption of the perspective camera model or the orthographic projection camera model. The quantified uncertainty components and estimates are merely illustrative, reflecting values which are expected to have in a sewer or drain CCTV inspection scenario. Therefore, in a real case scenario, the presented probabilistic formulation and quantification must be confirmed and updated if required.

In the study using the perspective camera model and considering the typical inspection scenario in drain and sewer system, a reduced focal distance lens is generally adopted, in order to have a large field-of-view. In this type of lens, distortion can cause the image geometrical deformation, thus affecting the accuracy of the performed dimensional measurements. Distortion coefficients and other relevant camera intrinsic parameters such as the focal distance and the principal point coordinates can be obtained, for example, from the DLT algorithm [15] implemented by a Matlab ${ }^{\circledR}$ toolbox [23] using images of traceable reference dimensional patterns.

The lens distortion model [24] included in this toolbox [23] has radial distortion components, $\Delta x_{\mathrm{rad}}$ and $\Delta y_{\mathrm{rad}}$, given by

$\Delta x_{\text {rad }}=\left(x-u_{0}\right) \cdot\left(k_{1} \cdot r^{2}+k_{2} \cdot r^{4}\right)$

$\Delta y_{\mathrm{rad}}=\left(y-v_{0}\right) \cdot\left(k_{1} \cdot r^{2}+k_{2} \cdot r^{4}\right)$

and tangential distortion components, expressed by

$\Delta x_{\tan }=p_{1} \cdot\left[r^{2}+2 \cdot\left(x-u_{0}\right)^{2}\right]+2 \cdot p_{2} \cdot\left(x-u_{0}\right) \cdot(y-$

$\left.v_{0}\right) \Delta x_{\tan }=p_{1} \cdot\left[r^{2}+2 \cdot\left(x-u_{0}\right)^{2}\right]+2 \cdot p_{2} \cdot\left(x-u_{0}\right) \cdot$

$\left(y-v_{0}\right)$

$\Delta y_{\tan }=2 \cdot p_{1} \cdot\left(x-u_{0}\right) \cdot\left(y-v_{0}\right)+p_{2} \cdot\left[r^{2}+2 \cdot(y-\right.$ $\left.\left.v_{0}\right)^{2}\right]$

where

$r=\sqrt{\left(x-u_{0}\right)^{2}+\left(y-v_{0}\right)^{2}}$.

Table 1 shows an example of the intrinsic parameters estimates and standard uncertainties obtained for the case of a camera with a $4 \mathrm{~mm}$ nominal focal distance lens and an image sensor with $480 \times 640$ squared pixels, considering a pixel linear dimension equal to $6.5 \mu \mathrm{m}$. High-order radial distortion coefficients were considered negligible. The standard uncertainty related to the image coordinates, resulting from the performed intrinsic parameterization, was equal to 0.04 pixel.

In order to know the impact of distortion in the image coordinate measurement accuracy, a Monte Carlo method was used [21], considering the results shown in Table 1 and the mathematical models given by expressions (16-19). The computational simulation algorithm was developed in Matlab ${ }^{\circledR}$, using the Mersenne-Twister pseudo-random number generator [25] and validated computational routines. $10^{5}$ trials were used in order to achieve convergent solutions.

Figure 6 shows the estimates of the image variation (in pixels) due to the combined effect of radial and tangential distortions, while Figure 7 presents the corresponding 95\% expanded measurement uncertainty.

As shown in Figure 6 and Figure 7, the distortion impact in the image coordinates is quite low. As expected, a higher distortion is observed in the extreme regions of the image, especially in the corners. The maximum distortion estimate is close to 0.050 pixel with a $95 \%$ expanded uncertainty of 0.001 pixel.

Table 1. Results obtained from the camera intrinsic parameterization.

\begin{tabular}{|c|c|c|c|}
\hline Input quantity & Symbol & Estimate & Standard uncertainty \\
\hline Focal distance & $f$ & $4.2739 \mathrm{~mm}$ & $0.0016 \mathrm{~mm}$ \\
\hline Principal point $x$-coordinate & $x_{0}$ & 302.72 pixel & 0.20 pixel \\
\hline Principal point y-coordinate & $y_{0}$ & 242.33 pixel & 0.19 pixel \\
\hline $1^{\text {st }}$ radial distortion coefficient & $k_{1}$ & $-891.7 \cdot 10^{-12}$ pixel $^{-2}$ & $2.5 \cdot 10^{-12}$ pixel $^{-2}$ \\
\hline $2^{\text {nd }}$ radial distortion coefficient & $k_{2}$ & $96.6 \cdot 10^{-17}$ pixel $^{-4}$ & $2.6 \cdot 10^{-17}$ pixel $^{-4}$ \\
\hline $1^{\text {st }}$ tangential distortion coefficient & $p_{1}$ & $-6.5 \cdot 10^{-10}$ pixel $^{-2}$ & $9.3 \cdot 10^{-10}$ pixel $^{-2}$ \\
\hline $2^{\text {nd }}$ tangential distortion coefficient & $p_{2}$ & $12.0 \cdot 10^{-11}$ pixel-2 $^{-2}$ & $9.3 \cdot 10^{-11}$ pixel $^{-2}$ \\
\hline
\end{tabular}




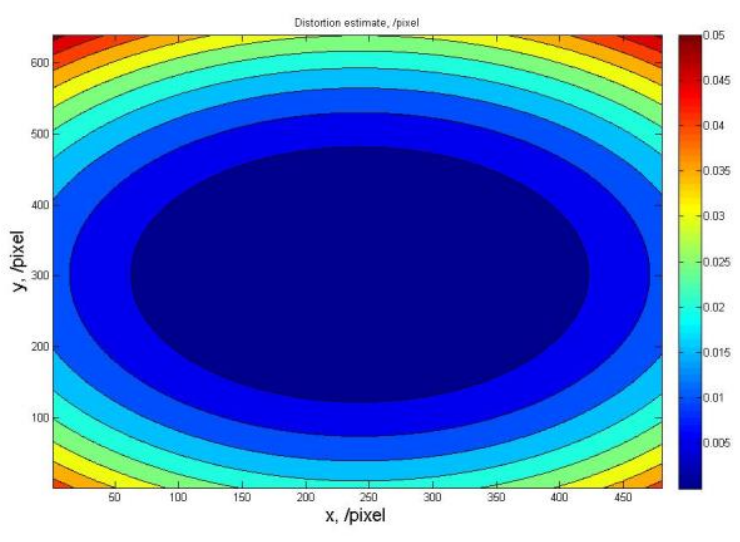

Figure 6. Image distortion estimates in pixels.

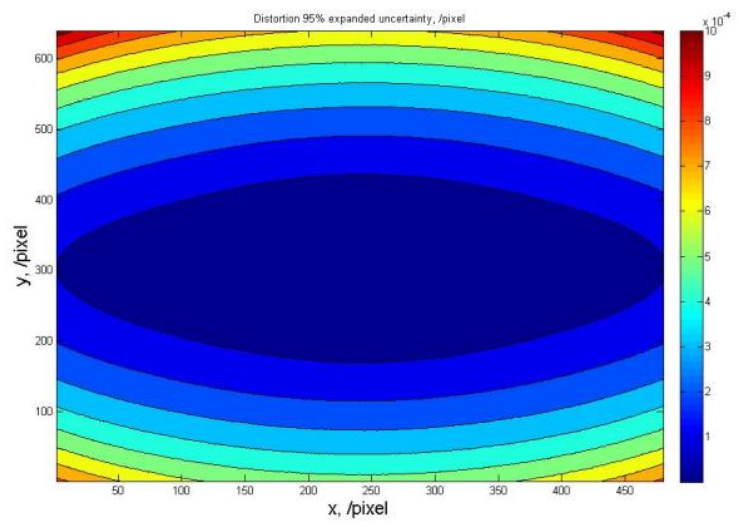

Figure 7. Image distortion 95\% expanded uncertainties in pixels.

These results allow the use of the collinearity equations (1-11) in the calculation of the dimensional measurement uncertainty, when considering the perspective camera model. With the exception of the distortion coefficients results shown in Table 1 , the remaining intrinsic parameters results were considered, however, three sets of unknown input variables are identified: (i) the camera's orientation angles estimates and measurement uncertainties; (ii) the camera's local coordinates and the location of the observation plane; (iii) the measurement uncertainty of the image coordinates.

This situation led to the adoption of the following assumptions: (i) the camera's orientation angles estimates and measurement uncertainties are available from the laboratorial

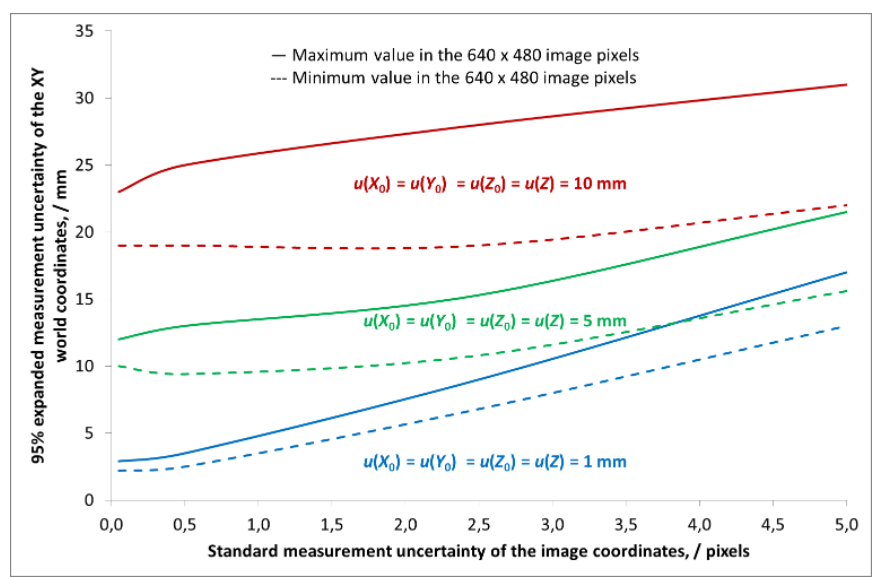

Figure 8. XY measurement uncertainties in the perspective camera model.

parameterization using the Matlab ${ }^{\circledR}$ toolbox [23] and can be used as a suitable approximation for an inspection scenario study; (ii) the camera is located in the local coordinate system origin (the centre of the drain or sewer cross-section), and the observation plane - parallel to the XY plane - is located $1 \mathrm{~m}$ away; the corresponding standard measurement uncertainties related to these estimates are considered equal and varying between $1 \mathrm{~mm}$ and $100 \mathrm{~mm}$; (iii) the measurement uncertainty of the image coordinates is comprised between 0.005 pixel (best case scenario, which only includes the uncertainty components related to the parameterization and distortion effects) and 5 pixels (worst case scenario, accounting for additional uncertainty components related to illumination and manual selection of interest points).

Table 2 summarizes the probabilistic formulation of the collinearity equations input quantities, which was used in the dimensional measurement uncertainty evaluation. Due to the non-linear and complex mathematical models related to perspective camera model (expressions 1 to 11), a Monte Carlo method [21] was again applied in the performed numerical simulations, in order to obtain the dispersion of values related to the $X$ and $Y$ local coordinates. A 95\% computational accuracy level lower than $1 \mathrm{~mm}$ was obtained.

Figure 8 shows the variation of the $95 \%$ expanded measurement uncertainty of the calculated $X$ and $Y$ local coordinates, considering the adopted measurement uncertainty intervals related to the standard uncertainty of the camera, observation plane location and image coordinates. The results obtained for a camera and plane location standard uncertainty above $5 \mathrm{~mm}$ are not shown due to the high value of the obtained

Table 2. Probabilistic formulation adopted for the input quantities related to the collinearity equations.

\begin{tabular}{|c|c|c|c|c|}
\hline Input quantity & Symbol & Probability density function & Estimate & Standard uncertainty \\
\hline Focal distance & $f$ & Gaussian & $4.2739 \mathrm{~mm}$ & $0.0016 \mathrm{~mm}$ \\
\hline Principal point $x$-coordinate & $x_{0}$ & Gaussian & 302.72 pixel & 0.20 pixel \\
\hline Principal point $y$-coordinate & $y_{0}$ & Gaussian & 242.33 pixel & 0.19 pixel \\
\hline \multirow[t]{3}{*}{ Camera orientation angles } & $\omega$ & Gaussian & $-0.0067 \mathrm{rad}$ & $0.0009 \mathrm{rad}$ \\
\hline & $\varphi$ & $\begin{array}{l}\text { Gaussian } \\
\text { Gaussian }\end{array}$ & $-0.0045 \mathrm{rad}$ & $0.0005 \mathrm{rad}$ \\
\hline & $\kappa$ & Gaussian & $0.0035 \mathrm{rad}$ & $0.0001 \mathrm{rad}$ \\
\hline \multirow{3}{*}{$\begin{array}{l}\text { Camera location in the global or local } \\
\text { coordinate system }\end{array}$} & $x_{0}$ & Gaussian & $0 \mathrm{~mm}$ & $1 \mathrm{~mm}-100 \mathrm{~mm}$ \\
\hline & $Y_{0}$ & Gaussian & $0 \mathrm{~mm}$ & $1 \mathrm{~mm}-100 \mathrm{~mm}$ \\
\hline & $Z_{0}$ & Gaussian & $0 \mathrm{~mm}$ & $1 \mathrm{~mm}-100 \mathrm{~mm}$ \\
\hline Observation plane location & $Z$ & Gaussian & $1000 \mathrm{~mm}$ & $1 \mathrm{~mm}-100 \mathrm{~mm}$ \\
\hline Image $x$-coordinate & $x$ & $\begin{array}{l}\text { Gaussian } \\
\text { Gaussian }\end{array}$ & 1 pixel -480 pixels & 0.005 pixel -5 pixels \\
\hline Image $y$-coordinate & $y$ & Gaussian & 1 pixel -640 pixels & 0.005 pixel -5 pixels \\
\hline
\end{tabular}




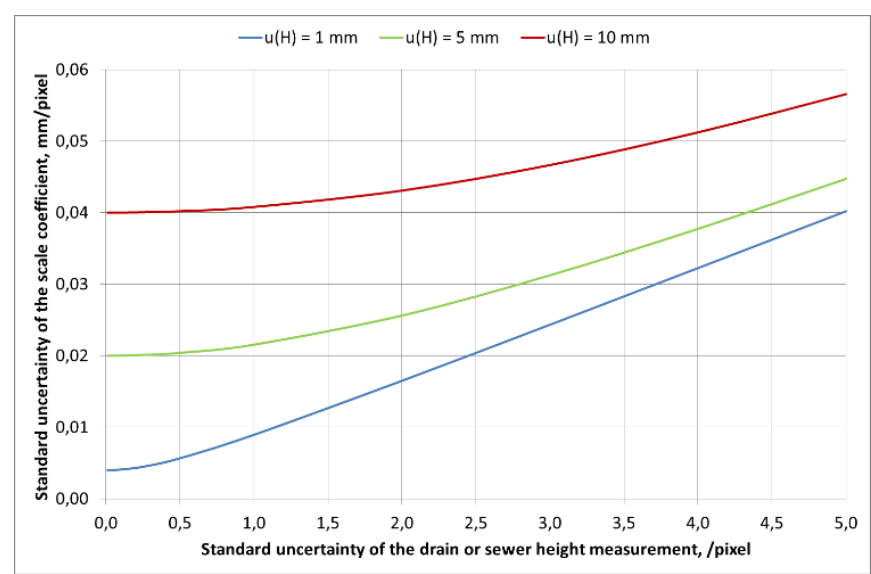

Figure 9. Standard uncertainty of the scale coefficient in the studied inspection scenario.

95\% expanded uncertainty of the $X$ and $Y$ coordinates (from 189 $\mathrm{mm}$ up to $263 \mathrm{~mm}$, for a $100 \mathrm{~mm}$ standard uncertainty).

The results show that a dimensional accuracy level lower than $10 \mathrm{~mm}$ can only be achieve for a camera and plane location standard uncertainty of $1 \mathrm{~mm}$ and an image coordinate standard uncertainty bellow 3 pixels. In a sensitivity point of view, the camera and plane standard uncertainty has a stronger contribution to the dimensional accuracy level, rather than the image coordinate measurement uncertainty.

When compared with the global dimensions of the corresponding camera field-of-view (974 $\mathrm{mm} \times 731 \mathrm{~mm})$, the relative $95 \%$ expanded measurement uncertainty of the $X$ and $Y$ coordinates, is comprised between $0.2 \%$ and $4.1 \%$, for the results shown in Figure 8.

In the study using the orthographic projection camera model, in a first stage, the measurement uncertainty of the scale coefficient is exemplified, considering an observation scenario where the drain or sewer height in the average cross-section plane is assumed to be $1000 \mathrm{~mm}$, corresponding to an image dimension of 500 pixels. The application of expression (12) originates an estimate equal to $2.00 \mathrm{~mm} /$ pixel.

Figure 9 illustrates the standard measurement uncertainty of the scale coefficient, considering different levels of measurement uncertainty related to the mentioned input quantities (from $1 \mathrm{~mm}$ up to $10 \mathrm{~mm}$, and from 0.01 pixel up to 5 pixels).

As seen in Figure 9, the standard measurement uncertainty of the scale coefficient can assume, in the considered inspection scenario, a minimum value of $0.004 \mathrm{~mm} /$ pixel and a maximum value of $0.057 \mathrm{~mm} /$ pixel.

These results can be propagated through expression (13) and combined with the previous considered standard uncertainty values of the dimensional measurement in the image (between 0.01 pixel and 5 pixels). Figure 10 presents the 95\% expanded relative uncertainty of dimensional measurements between $10 \mathrm{~mm}$ and $200 \mathrm{~mm}$.

In the worst case scenario, related to the scale coefficient with the highest measurement uncertainty (dashed lines in the plot shown in Figure 10), the obtained dimensional measurement accuracy is always above $5 \%$. Better accuracy levels are possible, namely, in the case of the lowest measurement uncertainty of the scale coefficient, for standard uncertainties of 1.3 pixel (for dimensional measurements close to $100 \mathrm{~mm}$ ) and 2.5 pixels (for dimensional measurements of $200 \mathrm{~mm}$ ).

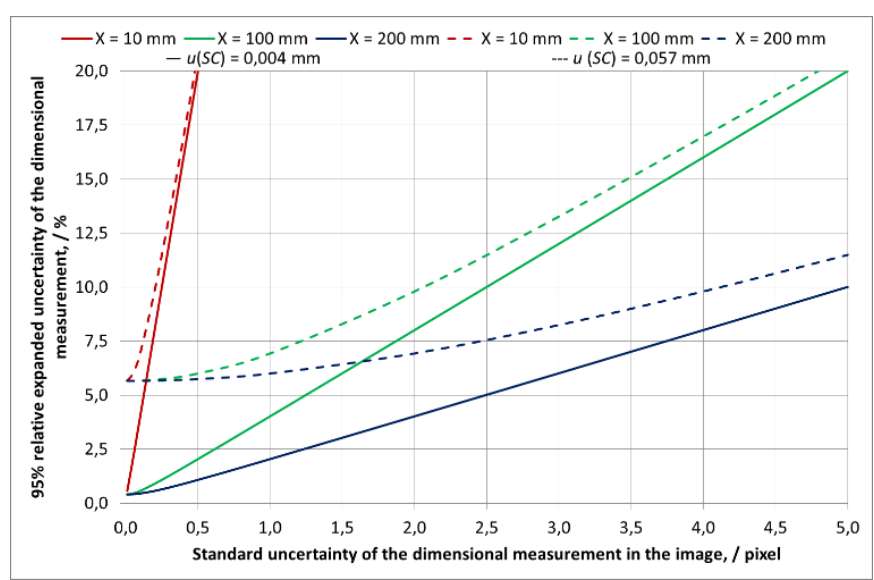

Figure 10. $95 \%$ relative expanded uncertainty of the dimensional measurement.

\section{CONCLUSIONS}

This paper presents the main contributions of a metrological perspective to the problem of the dimensional measurement accuracy in CCTV inspections, indicating experimental procedures applied in the optical characterization and modelling of the used cameras, followed by the corresponding suitable measurement uncertainty calculation procedure. This study gives a first step towards the improvement and the calculation of measurement accuracy in this field of inspection, namely, for the dimensional measurements obtained indirectly from the recorded inspection images.

Measurement uncertainty analysis tools were developed and are now available to be used within the approaches presented and for the type of experimental input information, regarding estimates and related measurement uncertainties. However, the results obtained so far in this study, already indicate a high impact of the accuracy of the camera and plane locations for the perspective camera model. In the orthographic projection camera model, results show a strong influence of the image quality in the accuracy of the dimensional measurements.

These calculation tools can also be used to quantify accuracy operational improvements, namely, due to changes in field lighting, image analysis, CCTV camera selection and metrological characterization.

Future work will be focused on determining the uncertainty component related to the adoption of the orthographic projection camera model, which is considered an approximation of the perspective camera model, and development of guidelines for operators, taking into consideration characteristics of available technologies.

\section{ACKNOWLEDGEMENT}

The authors acknowledge the financial support provided by LNEC - National Laboratory for Civil Engineering.

\section{REFERENCES}

[1] EN 752:2017, Drain and sewer systems outside buildings. Sewer system management, CEN - European Committee for Standardization, 2017.

[2] EN 13508-1:2012, Investigation and assessment of drain and sewer systems outside buildings - Part 1: General requirements, CEN - European Committee for Standardization, 2012. 
[3] EN 13508-2:2003+A1:2011, Investigation and assessment of drain and sewer systems outside buildings - Part 2: Visual inspection coding system, CEN - European Committee for Standardization, 2011.

[4] J. M. Makar, Diagnostic techniques for sewer systems, J. Infrastruct. Syst. 5 (1999) pp. 69-78.

[5] N. Stanic, M. Lepot, M. Catieau, J. Langeveld, F. Clemens, A technology for sewer pipe inspection (part 1): Design, calibration, corrections and potential application of a laser profiler, Automation in Construction 75 (2017) pp. 91-107.

[6] R. A. Fenner, Approaches to sewer maintenance: a review, Urban Water 2 (2000) pp. 343-356.

[7] T. C. Su, M. D. Yang, Application of morphological segmentation to leaking defect application in sewer pipelines, Sensors 14 (2014) pp. 8686-8704.

[8] T. C. Su, M. D. Yang, N. F. Pan, Y. F. Yang, Systematic image quality assessment for sewer inspection, Expert Syst. Appl. 38 (2011) pp. 1766-1776.

[9] D. Li, A. Cong, S. Guo, Sewer damage detection from unbalanced CCTV inspection using deep convolutional neural networks with hierarchical classification, Automation in Construction 10 (2019) pp. 199-208.

[10] A. Hawari, M, Alamin, F. Alkadour, M. Elmasry, T. Zayed, Automated defect detection tool for closed circuit television (CCTV) inspected sewer pipelines, Automation in Construction 89 (2018) pp. 99-109.

[11] B. Roghani, F. Cherqui, M. Ahmadi, P. Le Gauffre, M. Tabesh, Dealing with uncertainty in sewer condition assessment: Impact on inspection programs, Automation in Construction 103 (2019) pp. 117-126.

[12] T. P. Pridmore, D. Cooper, N. Taylor, Estimating camera orientation from vanishing point location during sewer surveys, Automation in Construction 5 (1997) pp. 407-419.

[13] J. Kannala, S. Brandt, J. Heikkilä, Measuring and modelling sewer pipes from video, Machine Vision and Applications 19 (2008) pp. $73-83$.
[14] P. Henley, Sewer condition - classification and training course, WRC, 2017.

[15] J. Heikkilä, O. Silvén, “A four-step camera calibration procedure with implicit image correction", Conference on Computer Vision and Pattern Recognition - Proceedings CVPR, 1997.

[16] R. Tsai, A versatile camera calibration technique for high-accuracy 3D machine vision metrology using off-the-shelf TV cameras and lenses, IEEE Journal of Robotics and Automation RA-3 4 (1987).

[17] Z. Zhang, A flexible new technique for camera calibration, IEEE Transactions on Pattern Analysis and Machine Intelligence 2211 (2000).

[18] EMVA 1288:2010, EMVA Standard 1288. Standard for Characterization of Image Sensors and Cameras, European Machine Vision Association, release 3.0, 2010.

[19] M. Rosenberger, C. Zhang, P. Votyakov, M. Peibler, R. Celestre, G. Notni, EMVA 1288 Camera characterisation and the influences of radiometric camera characteristics on geometric measurements, ACTA IMEKO 5, 4 (2016) pp. 81-87.

[20] Guide to the expression of Uncertainty in Measurements, ISO International Organization for Standardization, 1993, ISBN 9267-10188-9.

[21] Evaluation of measurement data - Supplement 1 to the "Guide to the expression of Uncertainty in Measurements" - Propagation of distributions using a Monte Carlo method, JCGM - Joint Committee for Guides in Metrology, 2008.

[22] R. Hartley, A. Zisserman, Multiple View Geometry in Computer Vision. Cambridge University Press, New York, 2003, ISBN 978 0521-54051-3.

[23] http://www.vision.caltech.edu/bouguetj/calib doc/

[24] D. Brown, "Close-range Camera Calibration", Proc. of the Symposium on Close-range Photogrammetry, 1971, Illinois, USA, pp. 855-866.

[25] M. Matsumoto, T. Nishimura, Mersenne twister: a 623dimensionally equidistributed uniform pseudo-random number generator, ACM Transactions on Modelling and Computer Simulation 81 (1998) pp. 3-30 